\title{
Cell-Intrinsic Roles for Autophagy in Modulating CD4 T Cell Functions
}

\author{
Elise Jacquin ${ }^{1,2 *}$ and Lionel Apeto ${ }^{1,2 *}$ \\ ${ }^{1}$ INSERM, U1231, Dijon, France, ${ }^{2}$ Université de Bourgogne Franche-Comté, Dijon, France
}

The catabolic process of autophagy plays important functions in inflammatory and immune responses by modulating innate immunity and adaptive immunity. Over the last decade, a cell-intrinsic role for autophagy in modulating CD4 T cell functions and differentiation was revealed. After the initial observation of autophagosomes in effector CD4 T cells, further work has shown that not only autophagy levels are modulated in CD4 T cells in response to environmental signals but also that autophagy critically affects the biology of these cells. Mouse models of autophagy deletion in CD4 T cells have indeed shown that autophagy is essential for CD4 T cell survival and homeostasis in peripheral lymphoid organs. Furthermore, autophagy is required for CD4 T cell

OPEN ACCESS

Edited by:

Amit Awasthi,

Translational Health Science and Technology Institute, India

Reviewed by:

Ashutosh Chaudhry,

Memorial Sloan Kettering Cancer Center, United States Cosima T. Baldari,

University of Siena, Italy

${ }^{*}$ Correspondence: Elise Jacquin elise.jacquin@inserm.fr; Lionel Apetoh lionel.apetoh@inserm.fr

Specialty section: This article was submitted to T Cell Biology, a section of the journal Frontiers in Immunology

Received: 30 January 2018 Accepted: 24 April 2018 Published: 09 May 2018

Citation:

Jacquin E and Apetoh L (2018) Cell-Intrinsic Roles for Autophagy in Modulating CD4 T Cell Functions.

Front. Immunol. 9:1023. doi: 10.3389/fimmu.2018.01023 proliferation and cytokine production in response to $T$ cell receptor activation. Recent developments have uncovered that autophagy controls CD4 T cell differentiation and functions. While autophagy is required for the maintenance of immunosuppressive functions of regulatory $T$ cells, it restrains the differentiation of $T_{H} 9$ effector cells, thus limiting their antitumor and pro-inflammatory properties. We will here discuss these findings that collectively suggest that therapeutic strategies targeting autophagy could be exploited for the treatment of cancer and inflammatory diseases.

Keywords: autophagy, T cell, CD4, differentiation, adaptive immunity, immunotherapy

\section{INTRODUCTION}

Macroautophagy, hereafter referred to as autophagy, is an evolutionary conserved catabolic pathway that ensures the degradation and recycling of intracellular components. During autophagy, cytosolic proteins and organelles are sequestered in a double membrane-bound structure called the autophagosome and eventually degraded upon fusion of the autophagosome with the lysosome (1). The autophagic flux accordingly refers to a complete catabolic process that ensures the breakdown of cargos and the release of the resulting macromolecules in the cytosol (2). Many autophagy-related (Atg) proteins have been found essential to orchestrate the formation of autophagosomes. This includes the upstream ULK complex (Ulk1/2, Rb1cc1/Fip200, Atg13, and Atg101) which regulates the induction of autophagosome formation. The class III phosphatidylinositol (PtdIns) 3-kinase (PIK3C3/Vps34) complex is then essential for the initial curvature of the phagophore and the recruitment of two ubiquitin-like conjugation systems (Atg4, 3/7/10, and 1611/5/12) which conjugate Atg8 homologs (microtubule-associated protein 1A/1B-light chain 3/LC3, GABARAPs) to phosphatidylethanolamine and thus ensure the elongation of the phagophore and its closure (Figure 1) $(1,3)$.

While autophagy was initially described as a nonselective process induced under various stress conditions including nutrient deprivation $(8,9)$, it is now clear that autophagy can also specifically target organelles, proteins, or pathogens for degradation. This selective autophagy process requires autophagy receptors such as p62/Sqstm1 (5). p62/Sqstm1 recognizes poly-ubiquitinated cargos 


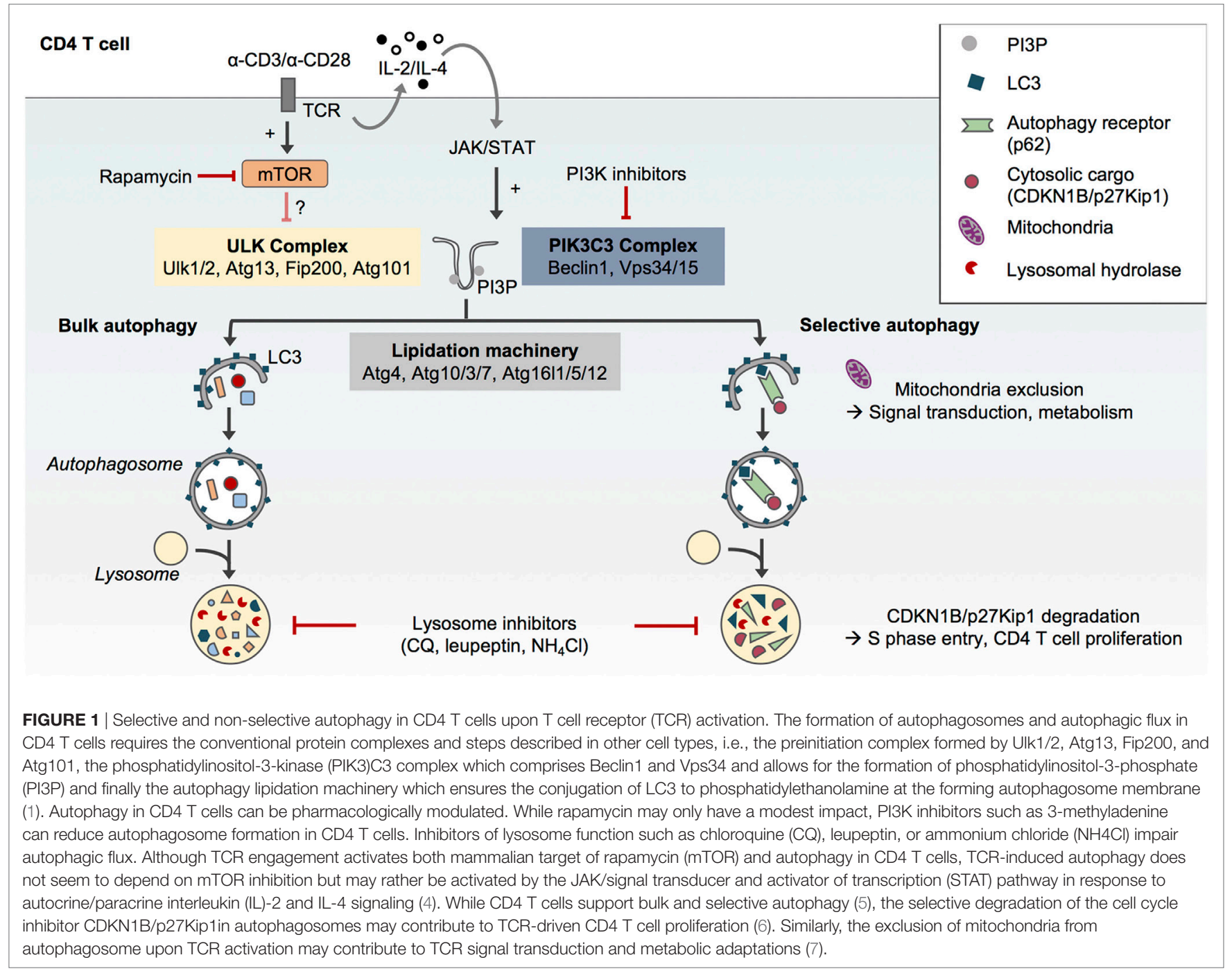

through its ubiquitin-associated domain and targets them for autophagic degradation through its LC3-interacting region motif (Figure 1). The levels of p62 are thus used as an index of autophagic degradation in combination with LC3 lipidation analysis (2).

Autophagy plays important functions in inflammatory and immune responses $(10,11)$. Atg proteins are crucial actors of cell-autonomous innate immunity. They contribute to pathogen elimination by antimicrobial selective autophagy (12) and LC3-associated phagocytosis (LAP), a recently identified form of noncanonical autophagy that directs LC3 lipidation onto endolysosomal compartments such as pathogen-containing phagosomes $(13,14)$. Moreover, both autophagy and LAP indirectly modulate adaptive immunity by contributing to antigen processing and major histocompatibility complex-restricted presentation to T cells (15). Over the last decade, accumulating evidence revealed a cell-intrinsic role for autophagy in CD4 T cell differentiation with direct consequences on physiopathology.

Here, work describing the regulation of autophagy in immune cells will be reviewed, with a focus on the functions of autophagy in CD4 $\mathrm{T}$ cell homeostasis and activation. We will also discuss recent findings showing that autophagy modulates the differentiation as well as the effector and regulatory functions of CD4 $\mathrm{T}$ cells and how this affects anticancer immune responses.

\section{AUTOPHAGY IS INDUCED IN CD4 T CELLS UPON ACTIVATION}

The first evidence of autophagosome formation in CD4 T cells was reported 10 years ago by $\mathrm{Li}$ and colleagues (16-18). The authors assessed the presence of double membrane-bound autophagosomes in mouse primary CD4 $\mathrm{T}$ cells differentiated in vitro into effector $\mathrm{T}$ helper $\left(\mathrm{T}_{\mathrm{H}}\right)$ cells. They focused on $\mathrm{T}_{\mathrm{H}} 1$ and $\mathrm{T}_{\mathrm{H}} 2$ cells, which are, respectively, essential for cell-mediated and humoral immunity (19). Using transmission electron microscopy, they detected autophagosomes in about $20 \%$ of $\mathrm{T}_{\mathrm{H}} 1$ and $\mathrm{T}_{\mathrm{H}} 2$ cells activated in vitro with anti-CD3 and anti-CD28 antibodies, whereas they did not observe autophagosome in naïve resting CD4 T cells. These findings were confirmed by the expression of exogenous green fluorescent protein (GFP)-LC3 fusion protein 
in effector T cells and monitoring of GFP-LC3 puncta formation by fluorescence microscopy. With this method, the authors measured the proportion of $\mathrm{T}_{\mathrm{H}} 1$ cells undergoing autophagy in various culture conditions and determined that $\mathrm{T}$ cell receptor (TCR) signaling can sustain autophagy in effector CD4 T cells (17). Shortly after, a study conducted by Pua and colleagues gave further support to these data by detecting increased levels of LC3 lipidation by Western blot in primary mouse CD4 T cells cultured in the presence of anti-CD3 antibodies (18). Accordingly, both reports showed for the first time that key autophagy genes Atg5, Atg7, Beclin1, and LC3 are expressed in CD4 T cells $(17,18)$. They also found that downregulation of the expression of these genes and inhibition of Jun amino-terminal kinase (JNK)/mitogen-activated protein kinase pathways or PtdIns-3kinase (PI3K) could inhibit autophagy in CD4 T cells, whereas the inhibition of mammalian target of rapamycin (mTOR) led to autophagy induction (17). These two initial reports, which evidenced that autophagy is induced in CD4 T cells upon TCR activation, were confirmed by several later studies conducted in mouse (4, 7, 20-22) and human primary CD4 T cells (23). In line with these studies, the expression of some autophagy proteins increases upon TCR activation. The activation of primary mouse CD4 T cells results in increased Beclin1 protein levels possibly after the activation of Becn 1 promoter by $\mathrm{p} 65 / \mathrm{NF}-\kappa \mathrm{B}$ (24). Upregulation of LC3 protein levels upon the activation of naive CD4 T cells and the reactivation of differentiated effector CD4 $\mathrm{T}$ cells has also been reported. Collectively, these studies indicate that the molecular mechanisms of autophagy in CD4 $\mathrm{T}$ cells are similar to those described in other cell types and that this pathway can be modulated by pharmacological and genetic approaches.

\section{MOLECULAR MECHANISMS REGULATING AUTOPHAGOSOME FORMATION IN CD4 T CELLS}

While TCR activation activates autophagosome formation in CD4 T cells, it has also been shown to induce mTOR activation (25). Botbol and colleagues have interrogated the involvement of mTOR in TCR-induced autophagy. To measure autophagic flux, the authors monitored LC3 lipidation in effector $\mathrm{T}_{\mathrm{H}} 1$ and $\mathrm{T}_{\mathrm{H}} 2$ cells cultured in various conditions in the presence of the inhibitors of lysosome function ammonium chloride $\left(\mathrm{NH}_{4} \mathrm{Cl}\right)$ and leupeptin. Surprisingly, effector $\mathrm{T}_{\mathrm{H}} 1$ and $\mathrm{T}_{\mathrm{H}} 2 \mathrm{CD} 4 \mathrm{~T}$ cells reactivated with anti-CD3 and anti-CD28 antibodies did not display an increased autophagic flux upon mTOR inhibition with rapamycin, suggesting that this process is mTOR-independent. However, it cannot be excluded that $\mathrm{T}_{\mathrm{H}} 1$ and $\mathrm{T}_{\mathrm{H}} 2 \mathrm{CD} 4 \mathrm{~T}$ cell reactivation on its own increased autophagic flux to its maximal level. This result may also suggest that TCR-induced autophagy signaling pathways other than mTOR can be involved in the regulation of autophagy in CD4 T cells such as the Janus tyrosine kinase (JAK)/ signal transducer and activator of transcription (STAT) signaling pathway. Indeed, the $\gamma$-chain cytokines interleukin (IL)-2 and IL-4, which are, respectively, produced by $\mathrm{T}_{\mathrm{H}} 1$ and $\mathrm{T}_{\mathrm{H}} 2$ cells upon reactivation, have been shown to contribute to autophagy induction in effector CD4 T cells in an autocrine/paracrine and JAK3-dependent manner (Figure 1) (4).

Data from the literature collectively suggest that autophagosome formation in CD4 T cells requires the canonical steps and molecules previously described in other cell types. For instance, overexpression of a kinase-dead mutant of the upstream autophagy protein ULK1 (ULK1 K461) in human naïve CD4 T cells impairs LC3 lipidation and autophagy (23). Likewise, reduced levels of autophagy have been described in CD4 T cells lacking the PI3K complex component Beclin1 (26) or the autophagy lipidation machinery proteins Atg7 (17, 18, 27), Atg3 (20), and Atg5 (28) (Figure 1). The requirement of PtdIns-3-phosphate (PI3P) formation by Vps34 during autophagy in CD4 T cells remains, however, elusive. Conditional deletion of Vps34 in mouse CD4 T cells does not completely suppress LC3 lipidation, and the PI3K inhibitor 3-methyladenine inhibits LC3 lipidation in Vps34-deficient cells, underscoring a possible contribution of Vps34-independent additional sources of PI3P in these cells (29-31). These findings can also indicate that noncanonical autophagy leading to endolysosomal LC3 lipidation may be active in CD4 T cells, a question that remains to be addressed.

Over the last decade, studies conducted in various models of autophagy deletion allowed for a better understanding of the molecular signals which control autophagy in CD4 T cells. These studies and others also strongly contributed to the understanding of the functions of autophagy in CD4 T cells. Indeed, while the early studies, which elegantly uncovered autophagosome formation in CD4 T cells, also suggested that autophagy could contribute to CD4 $\mathrm{T}$ cell death in vitro in response to specific stimuli $(16,17)$, they did not clearly ascribe a physiological function to autophagy in these cells.

\section{AUTOPHAGY MAINTAINS CD4 T CELL HOMEOSTASIS}

The first study aiming at exploring the role of autophagy in CD4 T cell biology was conducted by Pua and colleagues in 2007. The authors generated autophagy-deficient $\mathrm{T}$ cells by transferring Atg5-deficient fetal hematopoietic progenitors into lethally irradiated wild-type congenic hosts and investigated how autophagy contributes to T cell development and functions. The chimeric mice generated by Pua and colleagues displayed a reduced thymus cellularity as well as severely altered CD4 and CD8 T cell compartments in peripheral lymphoid organs. The authors attributed these results to the defective capacity of Atg $5^{-1-}$ lymphoid progenitors to reconstitute lymphoid compartments and the impaired survival of Atg5 $5^{-1-}$ CD8 and, to a lesser extent, CD4 T cells in the periphery (18). Since Atg5 was known to interact with proteins from the apoptosis pathway (32), Pua and colleagues generated a second autophagy-deficient mouse model. To overcome the possible effect of autophagy deletion on hematopoietic progenitor cell proliferation capacity, they

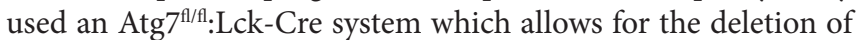
Atg7 from the double-positive stage of thymocyte development. Analyses of thymocytes and mature $\mathrm{T}$ cells revealed a decrease in CD4 and CD8 single-positive thymocyte numbers and a 
dramatic loss of CD4 and CD8 T cells in the peripheral lymphoid organs of $\operatorname{Atg} 7^{\mathrm{f} / \mathrm{f} l}:$ Lck-Cre animals, indicating a critical role for autophagy in maintaining peripheral CD4 T cell homeostasis (33). Along with lymphopenia in peripheral lymphoid organs, Pua and colleagues noted the acquisition of a "memory-like phenotype" by CD4 T cells characterized by a reduction in CD62L membrane levels and an increase in CD44 membrane levels (CD44 $4^{\text {high }} / \mathrm{CD} 62 \mathrm{~L}^{\text {low }}$ ) (33). These findings, which were further supported by several studies conducted in other models of autophagy deletion in mouse $\mathrm{T}$ cells, also demonstrated that autophagy is required for peripheral CD4 T cell survival (Table 1) $(7,20,28-31,34-36)$. Indeed, CD4 T cells isolated from Atg $7^{\mathrm{f} / \mathrm{fl}}$ : Lck-Cre mice displayed increased apoptosis levels as shown by Annexin $\mathrm{V}$ and active caspase staining together with an imbalance of anti- and pro-apoptotic proteins. Consistent with the role of autophagy in eliminating damaged organelles and protecting from cell death, Atg7-deficient CD4 T cells displayed an increased mitochondrial content and levels of reactive oxygen species (ROS) as well as an impaired regulation of mitochondrial number during their development (33). Supporting this idea, transcriptomic analyses conducted in Atg5-deficient thymocytes revealed an enrichment of transcripts encoding mitochondrion-associated proteins which can account for the increased mitochondrial mass observed in peripheral mature autophagy-deficient CD4 T cells (28). Furthermore, in vitro deletion of Atg3 in splenic T cells from $\operatorname{Atg} 3^{\mathrm{t} / / \mathrm{l}}$ estrogen receptor-Cre mice had no acute effect on organelle homeostasis and CD4 T cell survival but induced temporal accumulation of mitochondria and endoplasmic reticulum cell death after long-term culture. This suggested that the effect of autophagy deletion on peripheral CD4 T cell homeostasis is due to an accumulation of defects during their development rather than an acute phenomenon (20).
While further studies based on the deletion of Vps34 in CD4 $\mathrm{T}$ cells confirmed the critical role of autophagy in maintaining CD4 T cell survival during their development $(30,31)$, it was proposed that autophagy and autophagy proteins could favor CD4 T cell survival upon TCR stimulation. While Pua and colleagues initially suggested that in vitro TCR activation with anti-CD3 antibodies leads to the apoptotic cell death of Atg5-deficient CD4 $\mathrm{T}$ cells isolated from $\operatorname{Atg} 5^{-/-}$chimeric mouse splenocytes (18), further work conducted in mouse models of Atg7 deletion found similar levels of apoptosis in Atg7-competent and -deficient CD4 T cells upon in vitro TCR stimulation $(6,7)$. Conversely, targeting upstream components of the autophagy pathway seems to impair CD4 T cell survival upon TCR stimulation. Indeed, Kovacs and colleagues showed that the deletion of Beclin1 in CD4 T cells led to increased apoptosis upon TCR activation with anti-CD3 and anti-CD28 antibodies as indicated by increased levels of the pro-apoptotic genes Bim and pro-caspases 8 and 3 as well as DNA fragmentation. The authors proposed that the degradation of pro-caspase 8 by autophagy prevents its accumulation in protein complexes that function as signaling platforms to activate apoptosis. The ability of caspase inhibitor addition and modulation of pro- and antiapoptotic protein expression levels to prevent cell death further supported the apoptosis-mediated death of autophagy-deficient cells (34). Similarly, overexpression of a kinase-dead mutant of ULK1 in human CD4 T cells has been shown to induce mitochondria and ROS accumulation leading to CD4 T cell apoptosis upon TCR stimulation (23). It is worth noting that autophagy-independent functions Vps34 have also been shown to modulate CD4 T cell survival. Indeed, Vps34 deletion leads to CD4 T cell death, independently of autophagy and rather through impairment of trafficking and surface expression of IL-7 receptor, and regulation of IL-7 signaling (29).

TABLE 1 | Autophagy and CD4 T cell homeostasis.

\begin{tabular}{|c|c|c|c|c|c|c|c|}
\hline & \multirow[b]{2}{*}{$\begin{array}{l}\text { Mouse model of autophagy } \\
\text { deletion }\end{array}$} & \multicolumn{6}{|c|}{ Phenotypic observations } \\
\hline & & $\begin{array}{l}\text { Reduced SP } \\
\text { thymocyte } \\
\text { numbers }\end{array}$ & $\begin{array}{l}\text { Reduced } \\
\text { peripheral } \\
\text { CD4 T cell } \\
\text { numbers }\end{array}$ & $\begin{array}{l}\text { Memory-like } \\
\text { phenotype of } \\
\text { CD4 T cells }\end{array}$ & $\begin{array}{l}\text { Increased } \\
\text { CD4 T cell } \\
\text { apoptosis }\end{array}$ & $\begin{array}{c}\text { Increased } \\
\text { mitochondrial } \\
\text { content }\end{array}$ & Other observations \\
\hline Pua et al. (18) & Atg5 ${ }^{-/}$chimeras & $x$ & $x$ & & $x$ & & \\
\hline Pua et al. (33) & Atg $7^{f / 1 / 1}:$ Lck-Cre & $x$ & $x$ & $x$ & $x$ & $x$ & \\
\hline Stephenson et al. (28) & $\begin{array}{l}\text { Atg5 } 5^{f / / f l}: \text { Lck-Cre } \\
\text { Atg } 7^{f / / f l}: \text { Lck-Cre }\end{array}$ & $x$ & $X$ & $x$ & $x$ & $x$ & \\
\hline Hubbard et al. (7) & $\begin{array}{l}\text { Atg } 7^{\text {fl/1/1:}: L c k-C r e ~} \\
\text { Atg } 7^{\text {fl/f/1:}: E R-C r e ~}\end{array}$ & $x$ & $x$ & & & & \\
\hline Jia and $\mathrm{He}(20)$ & Atg $3^{1 / 1 / 1 /}:$ Lck-Cre & $x$ & $x$ & $x$ & $x$ & & \\
\hline Mcleod et al. (29) & Vps34 $4^{\text {f/fil }}:$ Lck-Cre & $x$ & $x$ & $x$ & $x$ & & \\
\hline Kovacs et al. (34) & Becn1 $1^{\mathrm{fl} / 1}:$ CD4-Cre & & $x$ & & & & \\
\hline Willinger and Flavell (30) & Vps34 ${ }^{\mathrm{f} / 1 / 1}:$ CD4-Cre & & $x$ & & & $x$ & $\begin{array}{l}\text { Increased CD8 T cell } \\
\text { apoptosis }\end{array}$ \\
\hline Parekh et al. (31) & Vps34 $4^{\mathrm{f} / \mathrm{fl}}: \mathrm{CD} 4-$ Cre & & $X$ & $x$ & $x$ & $x$ & \\
\hline Puleston et al. (35) & $\begin{array}{l}\text { Atg } 7^{f / f / f l}: \text { CD4-Cre } \\
\text { Atg5 } 5^{f / / f l}: \text { vav-Cre }\end{array}$ & & $x$ & X (in CD8 T cells) & & & $\begin{array}{l}\text { Impaired memory CD8 } \\
\mathrm{T} \text { cell formation }\end{array}$ \\
\hline Kabat et al. (36) & Atg1611 f/fil:CD4-Cre & & $x$ & $x$ & & & $\begin{array}{l}\text { Reduced peripheral } \\
\text { Foxp3 Treg cell } \\
\text { numbers }\end{array}$ \\
\hline
\end{tabular}

Phenotypic observations made from different mouse models of autophagy deficiency reported in the literature and identifying a role for autophagy in CD4 T cell homeostasis. SP, single positive; Lck, lymphocyte protein tyrosine kinase; ER, estrogen receptor; Cre, Cre recombinase; vav, vav guanine nucleotide exchange factor 1. 
By controlling activation-induced death of CD4 T cells but also their proliferation, differentiation, and cytokine production, TCR engagement is crucial for CD4 T cell homeostasis. Early after the first observation of autophagosomes in CD4 T cells, autophagy has not only been shown to be activated upon TCR engagement but also to modulate CD4 T cell responses to this signal.

\section{AUTOPHAGY CONTROLS CD4 T CELL PROLIFERATION IN RESPONSE TO TCR ACTIVATION}

Along with demonstrating the crucial role of autophagy for CD4 $\mathrm{T}$ cell homeostasis and survival in peripheral organs, Pua and colleagues also showed that autophagy induction following TCR activation promotes TCR-driven proliferation of CD4 T cells. Analysis of cell proliferation by 5(6)-carboxyfluorescein N-hydroxysuccinimidyl ester dilution revealed that Atg5deficient CD4 $\mathrm{T}$ cells display impaired proliferation following in vitro TCR activation with anti-CD3 antibodies, anti-CD28 antibodies, and IL-2. However, the normal levels of membrane TCR and activation markers CD69 and CD25 indicated that autophagy inhibition does not alter TCR-driven activation of CD4 T cells (18). While these findings were confirmed later in other mouse models of Atg3, Atg5, Atg7, and Vps34 deletion in CD4 T cells $(6,7,20,28,31)$, several groups aimed at uncovering the molecular mechanisms linking autophagy and CD4 T cell responses to TCR activation.

Jia and colleagues proposed that the selective degradation of the cell cycle inhibitor CDKN1B/p27Kip1 by p62-dependent autophagy may account for the TCR-driven proliferation defect observed in autophagy-deficient cells, as Atg3- and Atg7-deficient CD4 T cells accumulate CDKN1B/p27Kip1 and fail to enter S phase after in vitro TCR stimulation (Figure 1) (6). In an earlier study, the same group demonstrated that Atg7 deletion in CD4 $\mathrm{T}$ cells led to the accumulation of endoplasmic reticulum and impaired calcium mobilization upon in vitro TCR stimulation associated with an increased endoplasmic reticulum stress (37). However, this calcium mobilization defect does not seem to affect CD4 T cell activation since Atg7-deficient CD4 T cells display intact proximal TCR signaling and NF- $\kappa B$ pathway and maintain IL-2 production upon in vitro TCR activation (37).

The induction of autophagy upon TCR activation has also been proposed to regulate the energy metabolism changes required for $\mathrm{CD} 4 \mathrm{~T}$ cell activation. Indeed, in $\mathrm{T}_{\mathrm{H}} 1$ cells, both pharmacological and genetic inhibition of autophagy impair the production of ATP, interferon-gamma (IFN- $\gamma$ ), and IL-2 following TCR activation. These defects of autophagy-deficient $\mathrm{T}_{\mathrm{H}} 1$ cell functions can be reversed by the addition of methyl pyruvate, a cell-permeable glucose metabolism intermediate that can restore electron transport chain, and oxidative phosphorylation activity. Interestingly, the analysis of autophagosome content in $\mathrm{T}_{\mathrm{H}} 1$ cells revealed a change in autophagic cargos from mitochondria to soluble cytosolic component upon TCR activation (Figure 1). The authors proposed that this selective exclusion of mitochondria from autophagic degradation in response to TCR engagement may contribute to signal transduction and adaptation to energy requirement modification (7). Mitochondrion remodeling has been shown to control $\mathrm{T}$ cell metabolic adaptation, driving their response and fate following TCR activation, reinforcing the central role of this organelle in CD4 T cell biology (38).

A contrasting study conducted in effector $\mathrm{T}_{\mathrm{H}} 2$ cells has proposed that selective autophagy may prevent a sustained TCR activation by targeting the adaptor protein B-cell CLL/lymphoma 10 for degradation and thus limiting TCR-driven NF- $\mathrm{KB}$ activation. Nevertheless, this process does not seem to contribute to naive CD4 T cell response to TCR activation and has not been tested in other effector CD4 T cell subsets, suggesting that it may be limited to $\mathrm{T}_{\mathrm{H}} 2$ cells (39).

Collectively, these data demonstrated the importance of autophagy for CD4 $\mathrm{T}$ cell homeostasis not only during their development but also upon activation. These results are in line with increased levels of autophagy detected in the CD4 T cells of rheumatoid arthritis that may account for their hyperactivation and resistance to apoptosis (22). Until now, the specific role of autophagy in the different subtypes of effector and regulatory $\mathrm{T}$ cells has received less attention. Recent studies have shown that the function of autophagy may vary according to the subsets of CD4 T cells and most importantly that autophagy modulates CD4 $\mathrm{T}$ cell differentiation and functions by regulating energy metabolism or intracellular component levels.

\section{AUTOPHAGY REGULATES EFFECTOR AND REGULATORY CD4 T CELL DIFFERENTIATION AND FUNCTIONS}

In 2016, Wei and colleagues reported that autophagy is essential to maintain the functional integrity of the suppressor CD4 Foxp $3^{+}$ Treg cells (40), which suppress effector T cell functions $(41,42)$. The specific deletion of Atg7 in Foxp $3^{+}$CD4 $\mathrm{T}$ cells leads to reduced proportions of Foxp $3^{+} \mathrm{CD} 4 \mathrm{~T}$ cells in peripheral organs and a higher active caspase- 3 staining of the remaining cells, indicating a survival defect of autophagy-deficient Treg cells (40). The authors reported an increased proportion of Ki67-positive CD4 Treg cells purified from Atg $7^{\text {fl/fl }}$ :Foxp3Cre mice, probably reflecting a niche-filling behavior of the surviving cells. However, Atg7-deficient Treg cells proliferate normally in response to in vitro TCR stimulation with anti-CD3 antibodies, anti-CD28 antibodies, and IL-2 and after adoptive transfer into Rag $1^{-/-}$mice. This suggests that Atg7 may be dispensable for TCR-induced proliferation of Treg cells (40). Compared to autophagy-competent cells, Atg7-deficient Treg cells display an increased glycolytic activity upon TCR activation. This reveals an important role for autophagy in negatively regulating glucose metabolism in Treg cells by restraining the mTOR complex 1 (mTORC1)-c-Myc pathway. Indeed, the high mTORC1 activity observed in activated Atg7-deficient Treg cells and characterized by high phosphorylation levels of ribosomal protein S6 (S6) and eukaryotic translation factor $4 \mathrm{E}$ binding protein 1 (4EBP1) has been shown to rely on increased PI3K and pyruvate dehydrogenase kinase 1 abundance and activation and to be responsible for upregulated c-Myc expression and altered transcription programs (40). Importantly, autophagy controls transcriptional programs in Treg cells in a 
similar mTORC1-dependent manner. Atg7-deficient Treg cells display a reduced expression of Foxp3, Foxo, and Bach2, as well as an enrichment of effector T cell differentiation pathways that can be rescued by mTORC1 inhibition with rapamycin. This indicates a central role for autophagy in negatively regulating effector programs and maintaining Treg cell stability. These results are in line with the loss of Foxp3 expression and the aberrant production of IFN- $\gamma$ and IL-17 observed in Atg7-deficient Treg cells in vitro and in vivo (Figure 2). Moreover, autophagy is required for the ability of Treg cells to suppress antitumor responses in vivo as illustrated by an impaired tumor growth of MC38 colon adenocarcinoma cells in $\operatorname{Atg} 7^{\mathrm{A} / \mathrm{fl}}$ :Foxp3Cre mice associated with a loss of Treg cells and an increased expression of IFN- $\gamma$ from T cells at the tumor site. Although Atg7 deficiency also leads to survival defects of Treg cells, it is worth noting that autophagy seems to repress Treg cell apoptosis through a mechanism that does not solely rely on mTORC1 and which may thus be distinct from its role in Treg cell stability and functional integrity (40) (Figure 2).

In line with this, Le Texier and colleagues reported that the specific deletion of Atg7 in Foxp3 + CD4 T cells leads to the profound loss of the Helios + TIGIT + subset of Treg cells in the spleen and the bone marrow. The authors showed that autophagy is required for the survival of these Helios + TIGIT + Foxp3 + CD4 T cells and the maintenance of their immunosuppressive functions. Indeed, aged Atg $7^{\text {fl/fll}}$ :Foxp3Cre mice develop spontaneous $\mathrm{T}$ cell activation and multi-organ inflammation, suggesting an important role for autophagy-dependent Treg cells in the suppression of autoimmunity. Importantly, Treg-intrinsic autophagy promotes Treg reconstitution upon stem cell transplantation and attenuates graft versus host disease, supporting the idea that autophagydependent Treg cells are critical for tolerance (27).

In another model, the specific deletion of Atg16l1 in both CD4 T cells and Foxp $3{ }^{+} \mathrm{CD} 4 \mathrm{~T}$ cells results in a loss of Treg cells in mouse intestine and aberrant expression of the effector cytokines IFN- $\gamma$ and IL-17 by the remaining Treg cells, associated with $\mathrm{T}_{\mathrm{H}} 2$-driven intestinal inflammation toward dietary antigens and commensal microbiota (36). In this study, no evidence of defective in vitro Treg cell differentiation or stability was detected. Thus, Kabat and colleagues attributed the impairment of Atg16l1-deficient Treg cell survival to their aberrantly high glycolytic metabolism which prevents their metabolic adaptation to the intestinal mucosal environment. Indeed, the authors found that glycolysis gene expression levels are higher in Atg1611-deficient Treg cells compared to their autophagy-competent counterparts, especially
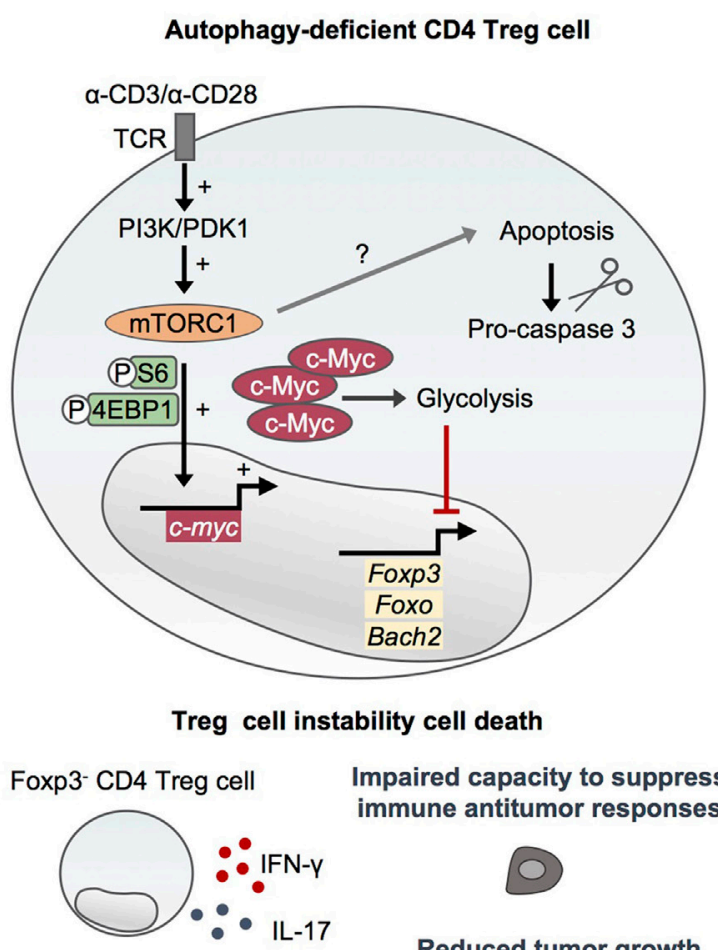

Impaired capacity to suppress immune antitumor responses

Reduced tumor growth

Autophagy-competent CD4 Treg cell

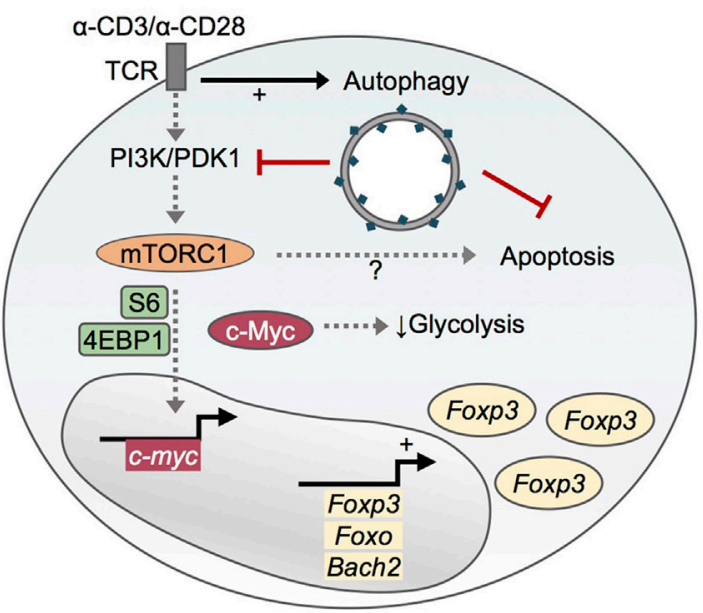

Treg cell maintenance

Foxp3+ CD4 Treg cell

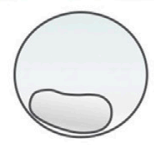

Tumor immune tolerance

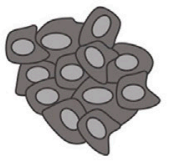

Increased tumor growth

FIGURE 2 | Autophagy maintains CD4 Treg cell stability and immunosuppressive functions. Activating environmental signals are essential for the functional maturation of CD4 Treg cells and activate both mammalian target of rapamycin (mTOR)C1 and autophagy. Autophagy-deficient CD4 Treg cells lacking Atg5 or Atg7 display an increased mTORC1 activity and upregulation of c-Myc and glycolytic metabolism (36, 40). This dysregulation of mTORC1/c-Myc pathway and energy metabolism leads to the loss of Foxp3, Foxo, and Bach2 which are essential for CD4 Treg cell differentiation and maintenance, and to an aberrant production of inflammatory cytokines (40). Furthermore, autophagy deficiency impairs CD4 Treg cell survival through a mechanism that seems to only partly rely on mTORC1 activity $(36,40)$. Autophagy thus plays a crucial role in maintaining the stability and functional integrity of CD4 Treg cells by restraining mTORC1-c-Myc pathway and glycolytic metabolism as well as promoting survival. Importantly, autophagy is required to maintain the ability of CD4 Treg cells to suppress antitumor immune responses in vivo (40) 
in Foxp $3^{+}$Treg cells sorted from the colon lamina propria of young Atg16l1-deficient mice. Furthermore, Treg cells differentiated in vitro from Atg1611-deficient naive CD4 T cells display higher expression levels of c-Myc and a panel of glycolysis genes, reduced expression levels of lipid metabolism-associated genes as well as higher rates of glycolysis and oxidative phosphorylation than autophagy-competent Treg cells, confirming that autophagy negatively controls glucose metabolism in Treg cells (36).

This study also revealed that autophagy deletion has an opposite effect on $\mathrm{T}_{\mathrm{H}} 2$ cells, promoting their expansion in peripheral tissues through both Treg cell-mediated control and cell-intrinsic mechanism. Glucose metabolism is not altered by autophagy deficiency in effector $\mathrm{T}_{\mathrm{H}} 2$ cells which display high levels of c-Myc and glycolysis gene expression as well as glycolytic energy metabolism, irrespective of their Atg1611 genotype (36), consistent with their high glycolytic rate compared to other CD4 T cell subsets (43). The constitutively high levels of glycolysis displayed by $\mathrm{T}_{\mathrm{H}} 2$ cells may allow them to adapt to the metabolic switch toward glycolysis induced by autophagy deficiency. GATA-3 having been previously associated with c-Myc-driven metabolic reprogramming leading to glycolysis induction upon TCR activation (44), the authors proposed that this transcription factor essential to $\mathrm{T}_{\mathrm{H}} 2$ cell differentiation may orchestrate the metabolic adaptations induced by autophagy deficiency. This suggests that the differential expression of transcription factors such as GATA-3 in the various subsets of regulatory and effector CD4 $\mathrm{T}$ cells may account for the different metabolic adaptations to autophagy deficiency (36). Together, these studies emphasize how the contribution of autophagy in energy metabolism varies according to the subsets of CD4 T cells, explaining, at least partly, the differential impact of autophagy deletion on CD4 T cell subset differentiation, stability, and functions.

In line with this, our recent study focused on the role of autophagy in the differentiation and functions of $\mathrm{T}_{\mathrm{H}} 9$ cells, characterized as IL-9 producing effector T cells which contribute to autoimmune diseases $(45,46)$ and exert potent anticancer functions (47-51). Using pharmacological and genetic approaches of autophagy inhibition in CD4 T cells, we showed that autophagy restrains $\mathrm{T}_{\mathrm{H}} 9$ cell differentiation and effector functions through a cell-intrinsic mechanism independent of energy metabolism modulation $(52,53)$. While we confirmed that mTORC1 activity is enhanced in Atg5-deficient in vitro differentiated Treg cells compared to autophagy-competent controls, we observed no difference in S6 and 4EBP1 phosphorylation in Atg5 ${ }^{-1-}$ CD4 $\mathrm{T}$ cells differentiated in vitro in effector $\mathrm{T}_{\mathrm{H}} 9$ cells, indicating that autophagy does not control mTORC1 signaling and thus glycolytic metabolism in $\mathrm{T}_{\mathrm{H}} 9$ cells. We proposed that $\mathrm{T}_{\mathrm{H}} 9$ cells display similar responses than $\mathrm{T}_{\mathrm{H}} 2$ cells regarding glycolytic metabolism in the absence of autophagy (53).

Although the inhibition of autophagy through genetic (Atg5 deletion in CD4 $\mathrm{T}$ cells) and pharmacological chloroquine approaches reduces the viability of in vitro differentiated effector and regulatory CD4 T cells, it specifically enhances IL-9 production and $\mathrm{T}_{\mathrm{H}} 9$ cell differentiation in vitro, without affecting the differentiation of other effector $\mathrm{T}$ cell subsets or skewing them toward $\mathrm{T}_{\mathrm{H}} 9$ differentiation program $(52,53)$. This role for autophagy in repressing IL-9 production by differentiating
$\mathrm{T}_{\mathrm{H}} 9$ cell relies on the degradation of PU.1, the master $\mathrm{T}_{\mathrm{H}} 9$ cell transcription factor (54), by selective autophagy. Indeed, upon $\mathrm{T}_{\mathrm{H}} 9$ cell differentiation, K63 ubiquitination of PU.1 leads to its specific recruitment by the autophagy receptor p62 via its ubiquitin-associated domain and the subsequent degradation of PU.1 in autophagosomes (Figure 3). $\mathrm{T}_{\mathrm{H}} 9$ cells treated with the inhibitor of lysosome function chloroquine (CQ) display increased IL-9 secretion and antitumor properties upon adoptive transfer. Indeed, CQ-treated in vitro differentiated $\mathrm{T}_{\mathrm{H}} 9$ cells have enhanced suppressive activity against melanoma tumor growth compared to $\mathrm{T}_{\mathrm{H}} 9$ cells displaying intact autophagic flux, suggesting that CQ may be considered in the context of adoptive T cell therapy of cancer $(52,53)$. Furthermore, genetic inhibition of autophagy in T cells leads to IL-9-dependent inhibition of tumor growth in mice bearing MC38 colon cancer and B16 melanoma and enhanced IL-9-producing CD4 tumor-infiltrating lymphocytes. While these results lend further support to the potent anticancer functions of $\mathrm{T}_{\mathrm{H}} 9$ cells (Figure 3), they suggest that autophagy induction in tumor microenvironment represses $\mathrm{T}_{\mathrm{H}} 9$ cell development and/or function, potentially explaining the low frequencies of $\mathrm{T}_{\mathrm{H}} 9$ cells detected in tumor lesions (48). This also suggests that inhibiting autophagy may restore antitumor immunity by concomitantly promoting $\mathrm{T}_{\mathrm{H}} 9$ cell-dependent antitumor immunity and impairing Treg cell stability (40), providing new opportunities for cancer immunotherapy.

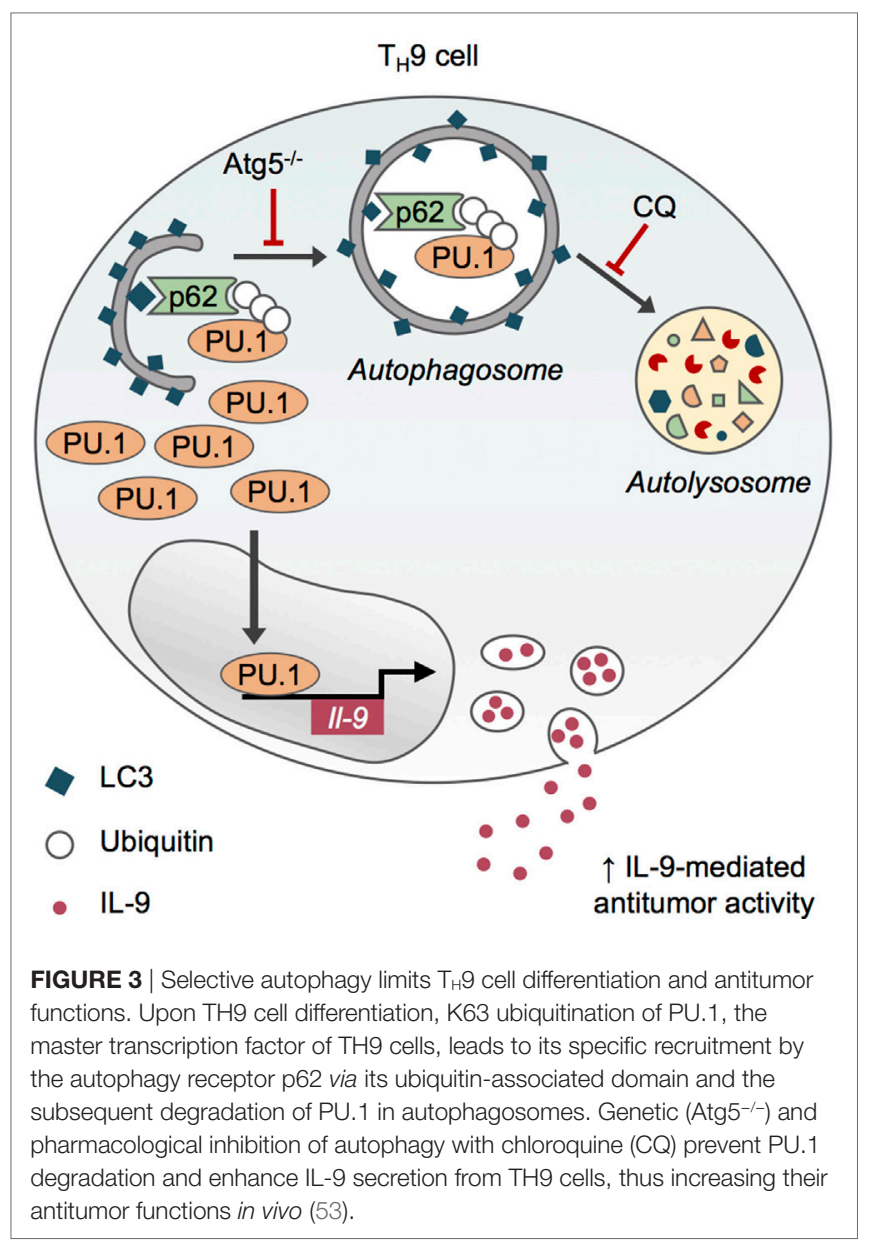


Through their pro-inflammatory functions, $\mathrm{T}_{\mathrm{H}} 9$ cells play an important role in the development of inflammatory bowel diseases (IBDs). Indeed, IL-9-secreting $\mathrm{T}_{\mathrm{H}} 9$ cells induce colitis in mice $(45,55)$, and PU.1-expressing cells as well as target cells expressing IL-9 receptor are frequently detected in the gut mucosa of IBD patients $(56,57)$. Autophagy contributes to inflammation, and IBD pathogenesis through multiple processes $(58,59)$ and mutations in Atg genes such as NOD2, ATG16L1, and ULK1 leading to autophagy deficiency have been associated with IBD susceptibility in humans (59). Our findings raise the hypothesis that autophagy may limit inflammation by repressing $\mathrm{T}_{\mathrm{H}} 9$ cells' inflammatory properties. Combined with the evidence that autophagy plays a central role in Treg cell-mediated immune tolerance in peripheral tissues $(36,40,60)$ and limits $T_{H} 2$ expansion in intestinal mucosa, they provide new insights that could be exploited for therapies against allergies as well as inflammatory and autoimmune diseases.

Collectively, investigators have demonstrated that autophagy levels in CD4 T cells are regulated in response to environmental signals and that autophagy controls CD4 T cell homeostasis and functions. The molecular mechanisms, which contribute to autophagy-driven modulation of effector and regulatory $\mathrm{T}$ cell functions, have also been clarified. The role for autophagy inhibition in restraining Treg cell functions and promoting $\mathrm{T}_{\mathrm{H}} 9$ cell differentiation evidenced in recent studies opens

\section{REFERENCES}

1. Feng Y, He D, Yao Z, Klionsky DJ. The machinery of macroautophagy. Cell Res (2014) 24:24-41. doi:10.1038/cr.2013.168

2. Klionsky DJ, Abdelmohsen K, Abe A, Abedin MJ, Abeliovich H, Acevedo Arozena A, et al. Guidelines for the use and interpretation of assays for monitoring autophagy (3rd edition). Autophagy (2016) 12:1-222. doi:10.1080/ 15548627.2015.1100356

3. Carlsson SR, Simonsen A. Membrane dynamics in autophagosome biogenesis. J Cell Sci (2015) 128:193-205. doi:10.1242/jcs.141036

4. Botbol Y, Patel B, Macian F. Common gamma-chain cytokine signaling is required for macroautophagy induction during CD4+ T-cell activation. Autophagy (2015) 11:1864-77. doi:10.1080/15548627.2015.1089374

5. Stolz A, Ernst A, Dikic I. Cargo recognition and trafficking in selective autophagy. Nat Cell Biol (2014) 16:495-501. doi:10.1038/ncb2979

6. Jia W, He MX, Mcleod IX, Guo J, Ji D, He YW. Autophagy regulates T lymphocyte proliferation through selective degradation of the cell-cycle inhibitor CDKN1B/p27Kip1. Autophagy (2015) 11:2335-45. doi:10.1080/15548627. 2015.1110666

7. Hubbard VM, Valdor R, Patel B, Singh R, Cuervo AM, Macian F. Macroautophagy regulates energy metabolism during effector $\mathrm{T}$ cell activation. J Immunol (2010) 185:7349-57. doi:10.4049/jimmunol.1000576

8. Yoshimori T. Autophagy: a regulated bulk degradation process inside cells. Biochem Biophys Res Commun (2004) 313:453-8. doi:10.1016/j.bbrc.2003. 07.023

9. Kroemer G, Marino G, Levine B. Autophagy and the integrated stress response. Mol Cell (2010) 40:280-93. doi:10.1016/j.molcel.2010.09.023

10. Levine B, Mizushima N, Virgin HW. Autophagy in immunity and inflammation. Nature (2011) 469:323-35. doi:10.1038/nature09782

11. Shibutani ST, Saitoh T, Nowag H, Munz C, Yoshimori T. Autophagy and autophagy-related proteins in the immune system. Nat Immunol (2015) 16:1014-24. doi:10.1038/ni.3273

12. Randow F. How cells deploy ubiquitin and autophagy to defend their cytosol from bacterial invasion. Autophagy (2011) 7:304-9. doi:10.4161/auto.7.3. 14539

13. Florey O, Kim SE, Sandoval CP, Haynes CM, Overholtzer M. Autophagy machinery mediates macroendocytic processing and entotic cell death by new therapeutic perspectives regarding the combination of autophagy inhibitors with anticancer immunotherapies. Recent cancer clinical trials have suggested that the clinically approved autophagy inhibitor hydroxychloroquine may be considered for cancer therapy although there is still a need for the development of more specific and potent autophagy inhibitors (61). Further work is now required to determine the effect of such treatments on CD4 T cell autophagy, differentiation, and functional integrity in vivo.

\section{AUTHOR CONTRIBUTIONS}

EJ and LA wrote the manuscript and EJ designed the figures.

\section{FUNDING}

The authors are supported by grants from the H2020 European Research Council (677251) (to LA) and the Fondation pour la Recherche Médicale (ARF20170938687) (to EJ). This work was supported by a French Government grant managed by the French National Research Agency under the program "Investissements d'Avenir" with reference ANR-11-LABX-0021 (Lipstic Labex), by the Conseil Régional de Bourgogne and the European Union through the PO FEDER-FSE Bourgogne $2014 / 2020$ programs.

targeting single membranes. Nat Cell Biol (2011) 13:1335-43. doi:10.1038/ ncb2363

14. Heckmann BL, Boada-Romero E, Cunha LD, Magne J, Green DR. LC3associated phagocytosis and inflammation. J Mol Biol (2017) 429:3561-76. doi:10.1016/j.jmb.2017.08.012

15. Munz C. Autophagy beyond intracellular MHC class II antigen presentation. Trends Immunol (2016) 37:755-63. doi:10.1016/j.it.2016.08.017

16. Espert L, Denizot M, Grimaldi M, Robert-Hebmann V, Gay B, Varbanov M, et al. Autophagy is involved in T cell death after binding of HIV-1 envelope proteins to CXCR4. J Clin Invest (2006) 116:2161-72. doi:10.1172/JCI26185

17. Li C, Capan E, Zhao Y, Zhao J, Stolz D, Watkins SC, et al. Autophagy is induced in CD4+ T cells and important for the growth factor-withdrawal cell death. J Immunol (2006) 177:5163-8. doi:10.4049/jimmunol.177.8.5163

18. Pua HH, Dzhagalov I, Chuck M, Mizushima N, He YW. A critical role for the autophagy gene Atg5 in T cell survival and proliferation. J Exp Med (2007) 204:25-31. doi:10.1084/jem.20061303

19. Mosmann TR, Cherwinski H, Bond MW, Giedlin MA, Coffman RL. Two types of murine helper T cell clone. I. Definition according to profiles of lymphokine activities and secreted proteins. J Immunol (1986) 136:2348-57.

20. Jia W, He YW. Temporal regulation of intracellular organelle homeostasis in T lymphocytes by autophagy. J Immunol (2011) 186:5313-22. doi:10.4049/ jimmunol.1002404

21. Matsuzawa Y, Oshima S, Takahara M, Maeyashiki C, Nemoto Y, Kobayashi M, et al. TNFAIP3 promotes survival of CD4 T cells by restricting mTOR and promoting autophagy. Autophagy (2015) 11:1052-62. doi:10.1080/15548627 .2015 .1055439

22. Van Loosdregt J, Rossetti M, Spreafico R, Moshref M, Olmer M, Williams GW, et al. Increased autophagy in CD4(+) T cells of rheumatoid arthritis patients results in T-cell hyperactivation and apoptosis resistance. Eur J Immunol (2016) 46:2862-70. doi:10.1002/eji.201646375

23. Watanabe R, Fujii H, Shirai T, Saito S, Ishii T, Harigae H. Autophagy plays a protective role as an anti-oxidant system in human $\mathrm{T}$ cells and represents a novel strategy for induction of T-cell apoptosis. Eur J Immunol (2014) 44:2508-20. doi:10.1002/eji.201344248

24. Copetti T, Bertoli C, Dalla E, Demarchi F, Schneider C. p65/RelA modulates BECN1 transcription and autophagy. Mol Cell Biol (2009) 29:2594-608. doi:10.1128/MCB.01396-08 
25. Pollizzi KN, Powell JD. Integrating canonical and metabolic signalling programmes in the regulation of T cell responses. Nat Rev Immunol (2014) 14:435-46. doi:10.1038/nri3701

26. Arsov I, Adebayo A, Kucerova-Levisohn M, Haye J, Macneil M, Papavasiliou FN, et al. A role for autophagic protein beclin 1 early in lymphocyte development. J Immunol (2011) 186:2201-9. doi:10.4049/jimmunol.1002223

27. Le Texier L, Lineburg KE, Cao B, Mcdonald-Hyman C, Leveque-El Mouttie L, Nicholls J, et al. Autophagy-dependent regulatory T cells are critical for the control of graft-versus-host disease. JCI Insight (2016) 1:e86850. doi:10.1172/ jci.insight. 86850

28. Stephenson LM, Miller BC, Ng A, Eisenberg J, Zhao Z, Cadwell K, et al. Identification of Atg5-dependent transcriptional changes and increases in mitochondrial mass in Atg5-deficient T lymphocytes. Autophagy (2009) 5:625-35. doi:10.4161/auto.5.5.8133

29. Mcleod IX, Zhou X, Li QJ, Wang F, He YW. The class III kinase Vps34 promotes $\mathrm{T}$ lymphocyte survival through regulating IL-7Ralpha surface expression. J Immunol (2011) 187:5051-61. doi:10.4049/jimmunol.1100710

30. Willinger T, Flavell RA. Canonical autophagy dependent on the class III phosphoinositide-3 kinase Vps34 is required for naive T-cell homeostasis. Proc Natl Acad Sci U S A (2012) 109:8670-5. doi:10.1073/pnas.1205305109

31. Parekh VV, Wu L, Boyd KL, Williams JA, Gaddy JA, Olivares-Villagomez D, et al. Impaired autophagy, defective $\mathrm{T}$ cell homeostasis, and a wasting syndrome in mice with a T cell-specific deletion of Vps34. J Immunol (2013) 190:5086-101. doi:10.4049/jimmunol.1202071

32. Maiuri MC, Zalckvar E, Kimchi A, Kroemer G. Self-eating and self-killing: crosstalk between autophagy and apoptosis. Nat Rev Mol Cell Biol (2007) 8:741-52. doi:10.1038/nrm2239

33. Pua HH, Guo J, Komatsu M, He YW. Autophagy is essential for mitochondrial clearance in mature T lymphocytes. J Immunol (2009) 182:4046-55. doi:10.4049/jimmunol.0801143

34. Kovacs JR, Li C, Yang Q, Li G, Garcia IG, Ju S, et al. Autophagy promotes T-cell survival through degradation of proteins of the cell death machinery. Cell Death Differ (2012) 19:144-52. doi:10.1038/cdd.2011.78

35. Puleston DJ, Zhang H, Powell TJ, Lipina E, Sims S, Panse I, et al. Autophagy is a critical regulator of memory CD8(+) T cell formation. Elife (2014) 3:e03706. doi:10.7554/eLife.03706

36. Kabat AM, Harrison OJ, Riffelmacher T, Moghaddam AE, Pearson CF, Laing A, et al. The autophagy gene Atg16l1 differentially regulates Treg and TH2 cells to control intestinal inflammation. Elife (2016) 5:e12444. doi:10.7554/ eLife. 12444

37. Jia W, Pua HH, Li QJ, He YW. Autophagy regulates endoplasmic reticulum homeostasis and calcium mobilization in T lymphocytes. J Immunol (2011) 186:1564-74. doi:10.4049/jimmunol.1001822

38. Buck MD, O'Sullivan D, Klein Geltink RI, Curtis JD, Chang CH, Sanin DE, et al. mitochondrial dynamics controls t cell fate through metabolic programming. Cell (2016) 166:63-76. doi:10.1016/j.cell.2016.05.035

39. Paul S, Kashyap AK, Jia W, He YW, Schaefer BC. Selective autophagy of the adaptor protein Bcl10 modulates $\mathrm{T}$ cell receptor activation of NF-kappaB. Immunity (2012) 36:947-58. doi:10.1016/j.immuni.2012.04.008

40. Wei J, Long L, Yang K, Guy C, Shrestha S, Chen Z, et al. Autophagy enforces functional integrity of regulatory $\mathrm{T}$ cells by coupling environmental cues and metabolic homeostasis. Nat Immunol (2016) 17:277-85. doi:10.1038/ni.3365

41. Sakaguchi S, Sakaguchi N, Asano M, Itoh M, Toda M. Immunologic selftolerance maintained by activated $\mathrm{T}$ cells expressing IL-2 receptor alphachains (CD25). Breakdown of a single mechanism of self-tolerance causes various autoimmune diseases. J Immunol (1995) 155:1151-64.

42. Hori S, Nomura T, Sakaguchi S. Control of regulatory T cell development by the transcription factor Foxp3. Science (2003) 299:1057-61. doi:10.1126/ science. 1079490

43. Michalek RD, Gerriets VA, Jacobs SR, Macintyre AN, Maciver NJ, Mason EF, et al. Cutting edge: distinct glycolytic and lipid oxidative metabolic programs are essential for effector and regulatory CD4+ T cell subsets. J Immunol (2011) 186:3299-303. doi:10.4049/jimmunol.1003613

44. Wan YY. GATA3: a master of many trades in immune regulation. Trends Immunol (2014) 35:233-42. doi:10.1016/j.it.2014.04.002

45. Dardalhon V, Awasthi A, Kwon H, Galileos G, Gao W, Sobel RA, et al. IL-4 inhibits TGF-beta-induced Foxp3+ T cells and, together with TGF-beta, generates IL-9+ IL-10+ Foxp3(-) effector T cells. Nat Immunol (2008) 9:1347-55. doi:10.1038/ni.1677

46. Veldhoen M, Uyttenhove C, Van Snick J, Helmby H, Westendorf A, Buer J, et al. Transforming growth factor-beta 'reprograms' the differentiation of $\mathrm{T}$ helper 2 cells and promotes an interleukin 9-producing subset. Nat Immunol (2008) 9:1341-6. doi:10.1038/ni.1659

47. Lu Y, Hong S, Li H, Park J, Hong B, Wang L, et al. Th9 cells promote antitumor immune responses in vivo. J Clin Invest (2012) 122:4160-71. doi:10.1172/ JCI65459

48. Purwar R, Schlapbach C, Xiao S, Kang HS, Elyaman W, Jiang X, et al. Robust tumor immunity to melanoma mediated by interleukin-9-producing $\mathrm{T}$ cells. Nat Med (2012) 18:1248-53. doi:10.1038/nm.2856

49. Vegran F, Berger H, Boidot R, Mignot G, Bruchard M, Dosset M, et al. The transcription factor IRF1 dictates the IL-21-dependent anticancer functions of TH9 cells. Nat Immunol (2014) 15:758-66. doi:10.1038/ni.2925

50. Abdul-Wahid A, Cydzik M, Prodeus A, Alwash M, Stanojcic M, Thompson M, et al. Induction of antigen-specific TH 9 immunity accompanied by mast cell activation blocks tumor cell engraftment. Int J Cancer (2016) 139:841-53. doi:10.1002/ijc.30121

51. Zhao Y, Chu X, Chen J, Wang Y, Gao S, Jiang Y, et al. Dectin-1-activated dendritic cells trigger potent antitumour immunity through the induction of Th9 cells. Nat Commun (2016) 7:12368. doi:10.1038/ncomms12368

52. Benoit-Lizon I, Jacquin E, Apetoh L. Selective autophagy restricts IL-9 secretion from TH9 cells: relevance in cancer growth. Cell Cycle (2018) 17(4):391-2. doi:10.1080/15384101.2017.1414680

53. Rivera Vargas T, Cai Z, Shen Y, Dosset M, Benoit-Lizon I, Martin T, et al. Selective degradation of PU.1 during autophagy represses the differentiation and antitumour activity of TH9 cells. Nat Commun (2017) 8:559. doi:10.1038/ s41467-017-00468-w

54. Chang HC, Sehra S, Goswami R, Yao W, Yu Q, Stritesky GL, et al. The transcription factor PU.1 is required for the development of IL-9-producing T cells and allergic inflammation. Nat Immunol (2010) 11:527-34. doi:10.1038/ ni. 1867

55. Gerlach K, Mckenzie AN, Neurath MF, Weigmann B. IL-9 regulates intestinal barrier function in experimental T cell-mediated colitis. Tissue Barriers (2015) 3:e983777. doi:10.4161/21688370.2014.983777

56. Gerlach K, Hwang Y, Nikolaev A, Atreya R, Dornhoff H, Steiner S, et al. TH9 cells that express the transcription factor PU.1 drive T cell-mediated colitis via IL-9 receptor signaling in intestinal epithelial cells. Nat Immunol (2014) 15:676-86. doi:10.1038/ni.2920

57. Nalleweg N, Chiriac MT, Podstawa E, Lehmann C, Rau TT, Atreya R, et al. IL-9 and its receptor are predominantly involved in the pathogenesis of UC. Gut (2015) 64:743-55. doi:10.1136/gutjnl-2013-305947

58. Martinez J, Cunha LD, Park S, Yang M, Lu Q, Orchard R, et al. Noncanonical autophagy inhibits the autoinflammatory, lupus-like response to dying cells. Nature (2016) 533:115-9. doi:10.1038/nature17950

59. Iida T, Onodera K, Nakase H. Role of autophagy in the pathogenesis of inflammatory bowel disease. World J Gastroenterol (2017) 23:1944-53. doi:10.3748/ wjg.v23.i11.1944

60. Tian Y, Liang X, Wu Y. The alternation of autophagy/apoptosis in CD4+CD25+Foxp3+ Tregs on the developmental stages of atherosclerosis. Biomed Pharmacother (2018) 97:1053-60. doi:10.1016/j.biopha.2017. 11.013

61. Chude CI, Amaravadi RK. Targeting autophagy in cancer: update on clinical trials and novel inhibitors. Int J Mol Sci (2017) 18:E1279. doi:10.3390/ ijms 18061279

Conflict of Interest Statement: The authors declare that the research was conducted in the absence of any commercial or financial relationships that could be construed as a potential conflict of interest.

Copyright (C) 2018 Jacquin and Apetoh. This is an open-access article distributed under the terms of the Creative Commons Attribution License (CC BY). The use, distribution or reproduction in other forums is permitted, provided the original author $(s)$ and the copyright owner are credited and that the original publication in this journal is cited, in accordance with accepted academic practice. No use, distribution or reproduction is permitted which does not comply with these terms. 